\title{
ADMINISTRACIÓN Y NEGOCIOS: JUNTOS PERO NO REVUELTOS
}

\author{
Thorstein Veblen, The theory of business enterprise, second \\ printing, New York, New American Library, 1963, 223 pp.
}

Guillermo Ruiz Pava*

$\mathrm{E}^{\mathrm{s}}$ s interesante que algunos fenómenos que hoy hacen parte del debate económico general se puedan entender de manera diferente a la luz de un desarrollo teórico de hace más de cien años. Este libro es uno de los más representativos del autor, eclipsado solo por La teoría de la clase ociosa (1899), y ambos son obras clásicas en la literatura de las ciencias sociales. Es, con toda propiedad, un aporte teórico no solo a la economía sino a las demás ciencias sociales que reconocen la interdependencia de los fenómenos sociales. Veblen estaba al tanto de las teorías económicas predominantes en su época y era hábil para encontrar sus relaciones. Aunque el alcance del libro puede ser demasiado amplio a mi parecer pues trata elementos del materialismo histórico junto con ideas de economistas como Marshall. E1 tono del libro es polémico, aun para nuestra época, pues buscaba romper esquemas, y quizá esa sea la razón del esfuerzo por integrar teorías y autores que parecen opuestos.

Dudo en calificar ese esfuerzo de infructuoso, aunque los objetivos teóricos fueran ambiciosos y el éxito sea difícil de establecer. Según el prefacio, el objetivo de la investigación es "la naturaleza, causas, utilidad y ulteriores tendencias de la empresa de negocios" y sus implicaciones para la estructura institucional (aunque Veblen no usa expresamente este término, sí ayuda a identificar en retrospectiva a lo que se refiere; no está de más recordar que es uno de los precursores de la economía institucional actual). En este sentido, reconoce que

* Magíster en Economía, estudiante de doctorado en Administración y miembro del grupo Historia y Empresariado de la Universidad de los Andes, Bogotá, Colombia, [ga.ruiz10@uniandes.edu.co]. Fecha de recepción: 5 de febrero de 2015, fecha de modificación: 14 de abril de 2015, fecha de aceptación: 29 de abril de 2015. Sugerencia de citación: Ruiz P., G. "Administración y negocios: juntos pero no revueltos", Revista de Economía Institucional 17, 32, 2015, pp. 405-410. DOI: 10.18601/01245996.v17n32.15 
el libro "peca” más por exceder los límites del análisis económico que por no abarcarlos en su totalidad.

El método histórico del análisis es evidente desde el principio, aunque es más preciso enmarcarlo en una perspectiva evolutiva. $\mathrm{Me}-$ todológicamente, en algunos apartes el análisis describe algunos fenómenos históricos y en otros es una explicación abstracta y ahistórica de ciertos hechos económicos; por esto es mejor llamarlo "evolutivo".

Veblen vivió entre 1857 y 1929 , cuando la sociedad estadounidense experimentaba grandes cambios que influyeron en su visión evolutiva. Pero si quisiéramos trazar paralelos entre el contexto histórico y su obra, tendría que hacerse con toda ella, de la cual La teoría de la empresa de negocios es solo una parte. Hay también secciones en las que emplea razonamientos lógico-deductivos enmarcados en la teoría económica predominante de su época.

En cuanto a la estructura del libro, hay planteamientos recurrentes $y$ se encuentran largos fragmentos que se refieren a temas tratados en capítulos anteriores. Los capítulos no tratan exclusivamente los temas a los que se refieren el título y los primeros párrafos; y a medida que se avanza en la lectura se analizan desde otros puntos de vista tópicos que se tratan en capítulos posteriores. Los capítulos se pueden agrupar de la siguiente manera: en primer lugar, los que definen, explican y analizan la empresa de negocios en todo su alcance; la introducción, el proceso mecánico (capítulo 2), la empresa de negocios (capítulo 3) y los principios de los negocios (capítulo 4). En segundo lugar, los que analizan las implicaciones de los préstamos y del crédito en la actividad regular de los negocios y en el proceso industrial; el uso del crédito (capítulo 5), el capitalismo comercial moderno (capítulo 6) y la teoría moderna del bienestar (capítulo 7). En tercer lugar, los que estudian las relaciones de la empresa de negocios con otras esferas de la sociedad; los principios comerciales en el derecho y la política (capítulo 8), la incidencia cultural del proceso mecánico (capítulo 9) y la decadencia natural de la empresa de negocios (capítulo 10).

\section{LA EMPRESA DE NEGOCIOS}

El análisis teórico empieza definiendo lo que Veblen llama "the machine process" (capítulos 2 y 3). Aunque la traducción literal sería el "proceso de la máquina”, es más apropiado traducirlo como el "proceso mecánico", un término que usa repetidamente en las primeras 40 páginas del libro, y que a veces sustituye por expresiones más familiares como "proceso industrial". Veblen describe con el mayor detalle posible el proceso de producción industrial y resalta algunas de sus 
características, como la estandarización, la coordinación permanente y la naturaleza física de la producción. Este proceso es "moderno" (p. 10), no surgió espontáneamente sino que se generó a lo largo del tiempo.

El proceso mecánico o industrial es diferente de las relaciones o transacciones de "negocios", y esta diferencia es esencial para entender su concepción teórica. Se trata de dos órdenes diferentes, con sus propia motivaciones, su propia lógica y, por supuesto, impulsados por actores diferentes. En el orden industrial, los ingenieros y los expertos técnicos se ocupan de hacer más eficientes los procesos de producción y los trabajadores, de cumplir los estándares de producción; la administración del proceso es el elemento esencial que permite la coordinación y los encadenamientos de las actividades industriales en toda la economía. En el orden comercial se encuentran las empresas, dirigidas por hombres de negocios con el fin de obtener ganancias "pecuniarias", que se obtienen en los intersticios del proceso industrial y no rara vez mediante el desajuste de los procesos industriales. Esta división refleja la postura crítica de Veblen ante el hombre de negocios, al que llamó "capitán de industria”:

Los hombres de negocios no crean las condiciones favorables para la consolidación industrial sobre esta base. Estos son asuntos del "estado de las artes industriales", y son el resultado del trabajo de los hombres que se dedican a empleos industriales y no de los que se ocupan de las actividades de negocios (p. 23).

Al comienzo, en sus etapas iniciales, los negocios y la industria estaban integrados debido al pequeño tamaño de los mercados. A medida que se expandió el comercio y las transacciones comerciales y de negocios se volvieron más frecuentes, en Europa y en Estados Unidos, a lo largo del siglo XIX la separación entre esos dos órdenes se hizo más evidente, hasta tal punto que las ganancias de los hombres de negocios no dependía unívocamente del ajuste eficiente del proceso industrial sino de las perturbaciones que podían ocasionar en los "intersticios" del proceso industrial, un hecho que era recurrente en los tiempos de Veblen. Los principios de los negocios que explican las relaciones entre la industria y las empresas de negocios se formulan en el capítulo 4 , pero se exponen en forma más sintética en el capítulo 7 , sobre la concepción moderna del bienestar:

1) La industria se desarrolla por medio de la inversión, que se realiza con miras a una ganancia pecuniaria $[\ldots]$

2) La industria a la que recurren los hombres de negocios como medio y forma de obtener ganancias tiene el carácter de un proceso mecánico [...] 
3) Estas relaciones pecuniarias intersticiales entre las diversas empresas o ramas de la industria que conforman el sistema industrial en su conjunto incluyen relaciones de crédito de mayor o menor duración [...]

4) Las dirección de la industria por empresas de negocios que compiten implica un uso extensivo del crédito (p. 91).

Así, en la economía moderna el proceso industrial está bajo la propiedad y el control de los hombres de negocios y funciona con el objetivo de obtener ganancias pecuniarias. El proceso industrial y las actividades de negocios dependen mutuamente, pero los negocios no podrían subsistir sin el "proceso mecánico" y el sistema de crédito.

\section{EL CRÉDITOY YOS CICLOS}

El análisis teórico de los ciclos, como fenómeno del orden de los negocios, se encuentra en los capítulos 5 a 8. Las notas de cada capítulo, que se incluyen al final del libro, son hasta ese momento una ayuda y un recurso aclaratorio. Antes aludían a los autores que se comentan en el texto, pero desde el capítulo 5, hasta el capítulo 7, algunas notas son un sustento formal (matemático, para ser más preciso) de lo que se argumenta en el cuerpo del texto. En este punto la lectura se vuelve difícil y lenta. Sugiero al lector que cuando llegue al capítulo 5, tome lápiz y papel y empiece a seguir el argumento junto con las fórmulas necesarias para entender el hilo conductor del argumento.

La argumentación se basa en una concepción del crédito en toda su extensión. Las consideraciones legales e institucionales relacionadas con los derechos de propiedad, un elemento vital del análisis, se posponen hasta el capítulo 8. Aunque quizá sea de mayor interés el examen de las implicaciones del uso de fondos prestables con garantías colaterales para la empresa de negocios, el proceso industrial y el bienestar de la sociedad. A mi modo de ver, para Veblen el uso del crédito (para él, las acciones son una forma de crédito, p. 49) explica las oscilaciones de los negocios y del proceso industrial en el mundo moderno. Un planteamiento que expone claramente en los capítulos 6 у 7 :

Pero, aparte de esos cambios incidentales que llevan la dirección de la industria a manos más competentes (o menos competentes), este uso competitivo de los fondos prestados no tiene ningún efecto agregado sobre los ingresos ni sobre el producto industrial (p. 51, las cursivas no son del original) ${ }^{1}$.

La cita anterior se refiere a lo que ocurre en el agregado cuando se expande el crédito. En mi interpretación, los términos que resalto en cursivas reflejan la noción clásica de que el dinero no crea riqueza.

${ }^{1}$ Las notas del capítulo 5, en particular las notas 5 y 17 , fundamentan el argumento formal de ese capítulo. 
No obstante, Veblen sostiene que la producción industrial puede incrementarse en forma indirecta cuando aumenta la intensidad del proceso industrial debido al estímulo generado por el crédito adicional, al que llama "efecto psicológico" (p. 52). Esta cita también muestra que Veblen hacía una distinción entre negocios y administración de la industria y la producción.

Esta reminiscencia de la ley de Say y de la concepción clásica contrasta con la visión más dinámica de los efectos del crédito sobre la economía que se plantea en el capítulo 7 . Los auges, las depresiones y las crisis son recurrentes en el mundo de los negocios o, en términos actuales, endógenos al sistema. E1 lector puede encontrar la descripción del ciclo de auges y crisis en las páginas 91-103. Una rápida síntesis del argumento es la siguiente: un incremento de la diferencia entre el precio y el costo, en una o en varias ramas de la industria, lleva a una expansión del crédito que permite a las empresas de negocios financiar la inversión en equipos y aumentar la capacidad industrial. Eventualmente, un grupo de empresas de una o varias ramas industriales no puede mantener ese diferencial entre precios y costos, bien sea por fallas de la administración, reducción de los precios o aumento de los costos. Esas empresas no pueden entonces cumplir con sus obligaciones, lo que lleva a una redistribución de la propiedad, en virtud de la caída del valor de las garantías colaterales de la deuda, y a la consiguiente pérdida de bienestar debida a la quiebra de empresas y al colapso del proceso industrial.

E1 "efecto psicológico" aludido anteriormente cumple un papel fundamental en la explicación de los efectos de las fluctuaciones del crédito sobre el proceso mecánico, aunque la relación de independencia entre producción agregada y crédito necesita mayor elaboración. Para Veblen, el efecto psicológico es un elemento central en una teoría de la industria, pero en una teoría de los negocios ocupa el lugar de un corolario (p. 52).

Estas ideas de Veblen suscitan muy diversas preguntas: ¿qué tanto aportan hoy las entidades financieras al producto real dado que las economías dependen mucho más del crédito y de instrumentos financieros que no existían en la época de Veblen? ¿Hasta qué punto el proceso de producción real depende hoy de los fondos prestables y cuál es su impacto en el agregado? ¿Qué determina la mayor capacidad de las empresas para mantener el diferencial entre precios y costos que da inicio al auge? ¿En qué medida la administración de los procesos productivos está a la merced de las ganancias inmediatas, de corto plazo, que desestabilizan el proceso industrial? 


\section{ULTIMOS CAPÍTULOS Y OBSERVACIONES}

Los últimos capítulos (8-10) extienden las consecuencias del orden de los negocios y del proceso mecánico, y de sus relaciones, a las instituciones legales, políticas y culturales. En principio, las instituciones legales garantizan los derechos de propiedad y facilitan el normal funcionamiento de los negocios. La política responde a la necesidad de ganancias pecuniarias y reacciona en forma agresiva (p. 185), hasta el punto de que puede afectar el curso de los negocios y el proceso industrial. Por su parte, la cultura se ve afectada por la estandarización y las pautas de eficiencia que caracterizan al proceso mecánico. Los hábitos antiguos de las personas se adaptan a las nuevas condiciones de estandarización y regularidad mecánica, aunque dependiendo del lugar que ocupan en la división social del trabajo, la clase social. Si bien en algunos apartes y en otras obras Veblen entra en mayores detalles, la siguiente cita muestra la diferenciación general entre las personas que se dedican a la producción industrial y las personas que se dedican a los negocios.

Pero aun después de haber hecho todas estas salvedades, es claro que la vida cotidiana de las clases dedicadas a los negocios difiere materialmente en el aspecto mencionado de la vida de las clases ocupadas en la industria propiamente dicha. Existe una apreciable y creciente diferencia entre los hábitos de vida de ambas clases; y esto provoca una creciente diferencia entre los hábitos de disciplina a los que están sujetas las dos clases (p. 151).

En este punto no parece distanciarse de Marx, al menos en apariencia, pues las prácticas y hábitos que tienen vigencia en una época histórica cambian, y surgen nuevos valores y costumbres consistentes con el proceso mecánico, o en palabras de Marx, con el desarrollo de las fuerzas productivas. La diferenciación de los dos órdenes de la sociedad moderna, uno ligado a los negocios y otro al proceso mecánico industrial, tiene un aire romántico mezclado con una actitud crítica hacia la clase que se ocupa de los negocios.

Esta obra es un gran esfuerzo por integrar un conjunto de teorías económicas y sociales para analizar el proceso industrial y las empresas de negocios, y ayuda a entender las crisis ocasionadas por la creciente expansión del crédito en las economías modernas. No obstante, el conjunto de fenómenos que intenta abarcar desborda los límites del análisis económico. 